\title{
Outage probability computation in multi-backscatter systems with multi-modes of operation
}

\author{
Dinh-Thuan Do, Anh-Tu Le \\ Faculty of Electronics Technology, Industrial University of Ho Chi Minh City, Ho Chi Minh City, Vietnam
}

\begin{abstract}
Article Info
Article history:

Received Jul 14, 2021

Revised Oct 19, 2021

Accepted Dec 29, 2021

\section{Keywords:}

Beamforming

Multi-backscatter devices

Non-orthogonal multiple

access

Outage probability

ABSTRACT

In this article, we study the outage performance of an ambient multi-backscatter system supported by non-orthogonal multiple access (NOMA) downlink transmission, where legacy users receive information either directly from the base station (BS) or from multiple backscatter devices (BDs) as well. Specifically, we characterize the network connections between the BS and network users into three modes of operation. In the first mode, the BS communicates with the users via the BDs by exploiting NOMA. In the second mode, the BS communicates directly with the users by employing selection combining (SC) beamforming. In the third mode, the users receive signals from both the BS and BDs simultaneously, by using maximal ratio combining (MRC) to combine the BS and BDs links at the users. Consequently, we derive the exact outage expressions of each mode and utilize Monte Carlo simulations to validate the individual exact expressions.
\end{abstract}

This is an open access article under the $\underline{C C B Y-S A}$ license.

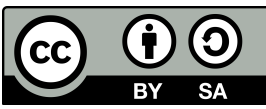

\section{Corresponding Author:}

Anh-Tu Le

Faculty of Electronics Technology, Industrial University of Ho Chi Minh City

12 Nguyn Văn Bo, Phng 4, Gò Vp, Thành ph H Chí Minh, Vietnam

Email: leanhtu@iuh.edu.vn

\section{INTRODUCTION}

The rapid deployment of internet-of-things (IoTs) in diverse systems such as energy systems (Smart Grid), healthcare systems, industrial systems, and transportation systems, has seen a tremendous push into researching ways of powering these billions of devices in a sustainable and environmentally friendly way [1], [2]. Ambient backscatter communication $(\mathrm{AmBC})$ has been proposed by the research community as a solution to address this unique challenge [3]. AmBCs can free up IoT devices from needing batteries as backscatter devices (BDs) harvest energy from traditional radio frequency (RF) transmitters such as television and radio transmitters to transmit information to nearby IoT devices [2]-[13]. Yang et al. in [4], the authors note that the harvested energy from ambient RF transmitters is adequate to provide power to high-rate battery-less sensors. Therefore, AmBC can be used in IoT environments where it's not convenient to replace or maintain sensor batteries, e.g., in wide-body area networks (WBANs) [14], [15]. However, due to the broadcast nature of ambient RF transmitters, AmBC tend to suffer from direct-link interference from the RF transmitter [4]-[6]. Several methods such as the design of maximum-likelihood (ML) detectors, and interference cancellation techniques, have been proposed to eliminate the direct-link interference, see [4], [6] and references therein for more details.

In this study, we propose utilizing non-orthogonal multiple access (NOMA) technology to address this problem, owing to its ability to simultaneously serve multiple wireless users over the same radio resource via linear superposition coding at the transmitter and successive interference cancellation (SIC) at the user's receiver [7], [16]-[18]. Therefore, integrating NOMA with AmBC networks shows great promise for future 
green IoT networks. Apart from NOMA, there have been several recent studies on deploying reconfigurable intelligent surfaces (RIS) to improve backscatter communication performance [19]-[21]. However, in this article, we focus on the contribution of NOMA to AmBCs performance. Our main contributions are: Firstly, we derive exact outage expressions for three practical AmBC link cases. In the first case, the base station (BS) communicates with the users via the BDs. In the second case, the BS communicates directly with the users. In the third case, the users receive signals from both the BS and BDs simultaneously; Secondly, from these expressions, we analyze the role of channel vectors, power allocation coefficients and backscatter reflection coefficient on outage probability.

All expressions are confirmed using Monte Carlo simulations. The paper is organized is being as. In section 2, we describe the proposed system model and signal characteristics at the users. Thereafter, in section 3 , we derive exact outage probability (OP) expressions depending on the mode of operation. In section 4, we explain results, followed by the summary in section 5 .

\section{RECEIVED SIGNALS AT TWO USERS}

In this paper, we study a downlink wireless system to allow the base station (BS) serving two multiple antennas users (user $U_{1}$ and user $U_{2}$ ) under assistance of multiple backscatter devices (BDs), shown in Figure 1. In some practical case, the second user $U_{2}$ might connect with the BS directly.

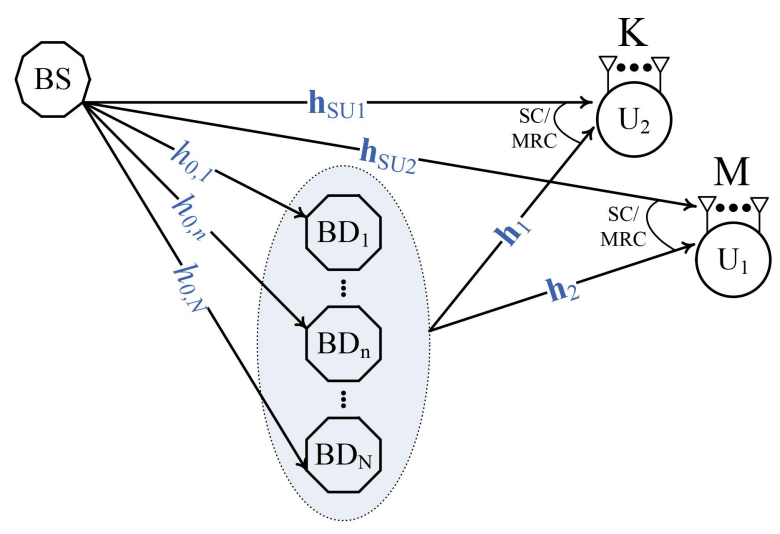

Figure 1. System model of multi-backscatter NOMA

The criteria to choose best multi-backscatter is given by:

$$
n^{*}=\arg \max _{n=1,2, \ldots, N}\left|h_{0, n}\right|^{2} \text {. }
$$

$B S$ broadcasts the information $x^{B S}$ to multi-backscatter $\left(B D_{n}\right), n=1,2, \ldots N$, which is given by:

$$
x^{B S}=\sqrt{a_{1} P_{S}} x_{1}+\sqrt{a_{2} P_{S}} x_{2},
$$

where $x_{1}$ and $x_{2}$ are the messages with unit power transmitted to $U_{1}$ and $U_{2}$, respectively. Further, $P_{S}$ is the total transmit power of the $B S$ with power allocation parameters $a_{1}$ and $a_{2}$, respectively, with $a_{1}>a_{2}$ and $a_{2}+a_{1}=1$.

The maximal-ratio combining (MRC) is used with beamforming vector to achieve optimal transmission scheme as in [22], [23] with $\left\|\mathbf{w}_{i}\right\|=1$ :

$$
w_{i}=\frac{h_{i}^{\dagger}}{\left\|h_{i}\right\|} \quad, i \in\{1,2\},
$$

where $\|\cdot\|$ denotes the Euclidean norm of a matrix.

The $B D$ the $B S$ signal to $U_{1}$ and $U_{2}$ with its own message $s$, where $\mathbb{E}\left\{|s|^{2}\right\}=1$. Thus, $U_{1}$ and $U_{2}$ receive one type of signal: the backscatter link signal from the $B D$. The received signals at $U_{1}$ and $U_{2}$ can thus be written as: 


$$
\begin{aligned}
& y_{U_{1}}=\beta h_{0, n^{*}}\left\|h_{1} w_{1}\right\| x^{B S} s+n_{U_{1}}, \\
& y_{U_{2}}=\beta h_{0, n^{*}}\left\|h_{2} w_{1}\right\| x^{B S} s+n_{U_{2}},
\end{aligned}
$$

where $\beta$ is a complex reflection coefficient used to normalize, $h_{1}$ and $h_{2}$ are the $1 \times M$ channel vector of $B D-U_{1}$ link and $1 \times K$ channel vector of $B D-U_{2}$ link, $n_{U_{1}}$ and $n_{U_{2}}$ are the additive white Gaussian noise (AWGN) with zero mean and variance matrix $\sigma_{U_{1}}^{2} I_{M}$ and $\sigma_{U_{2}}^{2} I_{K}$, respectively. Hence, the signal $x_{1}$ is detected successfully and $x_{2}$ as interference. Then, the signal-interference to ratio at $U_{1}$ is given by:

$$
\gamma_{1,1}=\frac{\rho a_{1} \beta^{2}\left\|h_{1}\right\|^{2}\left|h_{0, n^{*}}\right|^{2}}{\rho a_{2} \beta^{2}\left\|h_{1}\right\|^{2}\left|h_{0, n^{*}}\right|^{2}+1}
$$

where $\rho=\frac{P_{S}}{\sigma_{U_{i}}^{2}}, i \in\{1,2\}$ is the transmit signal-to-noise radio (SNR).

Thus, the instantaneous SINR at $U_{2}$ to detect $x_{1}$ and the instantaneous SNR at $U_{2}$ to detect $x_{2}$ is given respectively as:

$$
\gamma_{2,1}=\frac{\rho a_{1} \beta^{2}\left\|h_{2}\right\|^{2}\left|h_{0, n^{*}}\right|^{2}}{\rho a_{2} \beta^{2}\left\|h_{2}\right\|^{2}\left|h_{0, n^{*}}\right|^{2}+1}, \gamma_{2,2}=\rho a_{2} \beta^{2}\left\|\mathbf{h}_{2}\right\|^{2}\left|h_{0, n^{*}}\right|^{2}
$$

\section{OUTAGE PROBABILITY COMPUTATION OF DIFFERENT MODES}

\subsection{Scheme 1: outage probability for backscatter-non-orthogonal multiple access system without direct} link

In this study, we assume independent Rayleigh-distributed random variables (RVs) for all the channels. Thus, RVs $\left|h_{0, n^{*}}\right|^{2},\left\|h_{i}\right\|^{2}$ have exponential distributions, with $f_{\left|h_{0, n^{*}}\right|^{2}}(x)=\sum_{n=1}^{N}\left(\begin{array}{c}N \\ n\end{array}\right) \frac{n(-1)^{n-1}}{\Omega_{h_{0}}} e^{-\frac{n x}{\Omega_{h_{0}}}}$, $f_{\left\|\mathbf{h}_{i}\right\|^{2}}(x)=\frac{x^{N-1} e^{-\frac{x}{\Omega_{h_{i}}}}}{\Gamma(N) \Omega_{h_{i}}^{N}}$ and $F_{\left\|\mathbf{h}_{i}\right\|^{2}}(x)=1-e^{-\frac{x}{\Omega_{h_{i}}}} \sum_{p=0}^{P-1} \frac{x^{p}}{\Gamma(p+1) \Omega_{h_{i}}^{p}}$ with $\Gamma(x)=(x-1)$ ! is the gamma function.

The OP of $U_{1}$ is:

$$
\mathcal{O} \mathcal{P}_{U_{1}}^{\mathcal{I}}=\operatorname{Pr}\left(\gamma_{1,1}<\gamma_{1}\right)
$$

where $\gamma_{1}=2^{R_{1}}-1$ with $R_{1}$ being the target rate at $U_{1}$ to detect $x_{1}$.

Theorem 1. The exact OP expression of $U_{1}$ is calculated as:

$$
\begin{aligned}
\mathcal{O} \mathcal{P}_{U_{1}}^{\mathcal{I}}= & -2 \sum_{m=0}^{M-1} \sum_{n=1}^{N}\left(\begin{array}{c}
N \\
n
\end{array}\right) \frac{(-1)^{n-1} \theta_{1}^{m} n}{\Gamma(m+1) \Omega_{h_{1}}^{m} \Omega_{h_{0}}} \\
& \times\left(\frac{\theta_{1} \Omega_{h_{0}}}{\Omega_{h_{1}} n}\right)^{\frac{1-m}{2}} K_{1-m}\left(2 \sqrt{\frac{\theta_{1} n}{\Omega_{h_{1}} \Omega_{h_{0}}}}\right),
\end{aligned}
$$

where $\theta_{1}=\frac{\gamma_{1}}{\left(a_{1}-\gamma_{1} a_{2}\right) \rho}$. Note 9 is based on the condition of $a_{1}>\gamma_{1} a_{2}$

Proof:

$$
\begin{aligned}
\mathcal{O} \mathcal{P}_{U_{1}}^{\mathcal{I}} & =1-\operatorname{Pr}\left(\left\|h_{1}\right\|^{2}>\frac{\theta_{1}}{\beta^{2}\left|h_{0, n^{*}}\right|^{2}}\right) \\
& =1-\int_{0}^{\infty} \bar{F}_{\left\|h_{1}\right\|^{2}}\left(\frac{\theta_{1}}{\beta^{2} t}\right) f_{\left|h_{0, n^{*}}\right|^{2}}(t) d t \\
& =1-\sum_{m=0}^{M-1} \sum_{n=1}^{N}\left(\begin{array}{c}
N \\
n
\end{array}\right) \frac{(-1)^{n-1} \theta_{1}^{m} n}{\Gamma(m+1) \Omega_{h_{1}}^{m} \beta^{2 m} \Omega_{h_{0}}} \Phi(t),
\end{aligned}
$$


where $\bar{F}_{\left\|\mathbf{h}_{1}\right\|^{2}}(x)=1-F_{\left\|\mathbf{h}_{1}\right\|^{2}}(x)$ and $\Phi(t)=\int_{0}^{\infty} e^{-\frac{\theta_{1}}{\beta^{2} \Omega_{h_{1}}}-\frac{n t}{\Omega_{h_{0}}}} t^{-m} d t$.

Based on [24], (3.471.9). We can rewrite (10) as:

$$
\begin{aligned}
\mathcal{O} \mathcal{P}_{U_{1}}^{\mathcal{I}}= & -2 \sum_{m=0}^{M-1} \sum_{n=1}^{N}\left(\begin{array}{c}
N \\
n
\end{array}\right) \frac{(-1)^{n-1} \theta_{1}^{m} n}{\Gamma(m+1) \Omega_{h_{1}}^{m} \beta^{2} \Omega_{h_{0}}} \\
& \times\left(\frac{\theta_{1} \Omega_{h_{0}}}{\beta^{2} \Omega_{h_{1}} n}\right)^{\frac{1-m}{2}} K_{1-m}\left(2 \sqrt{\frac{\theta_{1} n}{\Omega_{h_{1}} \beta^{2} \Omega_{h_{0}}}}\right) .
\end{aligned}
$$

Next, the OP of $U_{2}$ is given by:

$$
\mathcal{O} \mathcal{P}_{U_{2}}^{\mathcal{I}}=\operatorname{Pr}\left(\gamma_{2,2}<\gamma_{2}\right)=1-\operatorname{Pr}\left(\left\|\mathbf{h}_{2}\right\|^{2}\left|h_{0, n^{*}}\right|^{2}>\theta_{2}\right),
$$

where $\gamma_{2}=2^{R_{2}}-1$ with $R_{2}$ being the target rate at $U_{2}$ to detect $x_{2}, \theta_{2}=\frac{\gamma_{2}}{\rho a_{2}}$. Similarly with solving $\mathcal{O P} \mathcal{P}_{U_{1}}^{\mathcal{I}}$ can be achieved $\mathcal{O} \mathcal{P}_{U_{2}}^{\mathcal{I}}$ as:

$$
\begin{aligned}
\mathcal{O} \mathcal{P}_{U_{2}}^{\mathcal{I}}= & -2 \sum_{k=0}^{K-1} \sum_{n=1}^{N}\left(\begin{array}{c}
N \\
n
\end{array}\right) \frac{(-1)^{n-1} \theta_{2}^{k} n}{\Gamma(k+1) \Omega_{h_{2}}^{k} \beta^{2 k} \Omega_{h_{0}}} \\
& \times\left(\frac{\theta_{2} \Omega_{h_{0}}}{\beta^{2} \Omega_{h_{2}} n}\right)^{\frac{1-k}{2}} K_{1-k}\left(2 \sqrt{\frac{\theta_{2}}{\Omega_{h_{2}} \beta^{2} \Omega_{h_{0}}}}\right) .
\end{aligned}
$$

\subsection{Scheme 2: outage probability of directed link by selection combining mode}

In the MRT scheme, to maximize the signal-to-interference noise (SINR) at $U_{1}$ and $U_{2}, B S$ uses a beamforming transmit vector $w_{r}=\frac{h_{r}^{\dagger}}{\left\|h_{r}\right\|}, r \in\left\{S U_{1}, S U_{2}\right\}$ to signal $x^{B S}$ before sending $w_{r}$ to users, $h_{S U_{1}}$ and $h_{S U_{2}}$ are the $1 \times M$ and $1 \times K$ channel vector of $B S$ to $U_{1}$ link and $B S$ to $U_{2}$ link, respectively. The $B S$ transmits signals directly to two users as

$$
y_{r}=\left\|h_{r} w_{r}\right\| x^{B S}+n_{r} \quad, r \in\left\{S U_{1}, S U_{2}\right\} .
$$

We have SINR at $U_{1}$ as:

$$
\gamma_{S U_{1}}=\frac{\left\|h_{S U_{1}}\right\|^{2} a_{1} \rho}{\left\|h_{S U_{1}}\right\|^{2} a_{2} \rho+1},
$$

and SINR and the successive interference cancellation (SIC) at $U_{2}$ as $\gamma_{S U_{2,1}}=\frac{\left\|h_{S U_{2}}\right\|^{2} a_{1} \rho}{\left\|h_{S U_{2}}\right\|^{2} a_{2} \rho+1}$ and $U_{2}$ as $\gamma_{S U_{2}}=\left\|h_{S U_{2}}\right\|^{2} a_{2} \rho$, respectively.

Finally, the instantaneous SINRs at $U_{1}$ and $U_{2}$ based on selection combining, can be written as:

$$
\gamma_{i}^{S C}=\operatorname{Pr}\left(\max \left(\gamma_{S U_{i}}, \gamma_{i, i}\right)<\gamma_{i}\right) \quad, i \in\{1,2\}
$$

The OP of $U_{1}$ is given by:

$$
\begin{aligned}
& F_{\gamma_{1,1}}(x)=\operatorname{Pr}\left(\left\|h_{1}\right\|^{2}<\frac{x}{\Delta(x) \mid h_{0,\left.n^{*}\right|^{2}}}\right) \\
& =1-\int_{0}^{\infty} \bar{F}_{\left\|\mathbf{h}_{1}\right\|^{2}}\left(\frac{x}{\Delta(x) t}\right) f_{\left|h_{0, n^{*}}\right|^{2}}(t) d t \\
& =1-\sum_{m=0}^{M-1} \sum_{n=1}^{N}\left(\begin{array}{c}
N \\
n
\end{array}\right) \frac{n x^{m}(-1)^{n-1}}{\Gamma(m+1) \Omega_{h_{1}}^{m} \Omega_{h_{0}} \Delta(x)^{m}} \delta(t \mid x),
\end{aligned}
$$

where $\delta(t \mid x)=\int_{0}^{\infty} e^{-\frac{x}{\Omega_{h_{1}} \Delta(x) t}-\frac{n t}{\Omega_{h}}} t^{-m} d t$ and $\Delta(x)=\left(a_{1}-x a_{2}\right) \rho \beta^{2}$. Using [24], (3.471.9) and after some transform. $F_{\gamma_{1,1}}(x)$ and $F_{\gamma_{S U_{2}}}(x)$ can be express respectively as:

$$
\begin{aligned}
& F_{\gamma_{1,1}}(x)=1-2 \sum_{m=0}^{M-1} \sum_{n=1}^{N}\left(\begin{array}{c}
N \\
n
\end{array}\right) \frac{n x^{m}(-1)^{n-1}}{\Gamma(m+1) \Omega_{h_{1}}^{m} \Omega_{h_{0}} \Delta(x)^{m}} \\
& \times\left(\frac{x \Omega_{h_{0}}}{\Omega_{h_{1}} \Delta(x)}\right)^{\frac{1-m}{2}} K_{1-m}\left(2 \sqrt{\frac{x n}{\Omega_{h_{1}} \Omega_{h_{0}} \Delta(x)}}\right),
\end{aligned}
$$


and,

$$
F_{\gamma_{S U_{1}}}(x)=1-\sum_{m=0}^{M-1} \frac{x^{m} e^{-\frac{x}{\left(a_{1}-x a_{2}\right) \rho \Omega_{S U_{1}}}}}{\Gamma(m+1) \Omega_{S U_{1}}^{m}\left(a_{1}-x a_{2}\right)^{m} \rho^{m}} .
$$

Substituting $\sqrt{18}$ and $\sqrt{19}$ into $\sqrt{16}$, , the close-form of OP of $\mathcal{O} \mathcal{P}_{U_{1}}^{\mathcal{I I}}$ is obtained as:

$$
\begin{aligned}
& \mathcal{O} \mathcal{P}_{U_{1}}^{\mathcal{I I}}=\left[1-2 \sum_{m=0}^{M-1} \sum_{n=1}^{N}\left(\begin{array}{c}
N \\
n
\end{array}\right) \frac{n \gamma_{1}^{m}(-1)^{n-1}}{\Gamma(m+1) \Omega_{h_{1}}^{m} \Omega_{h_{0}} n \Delta\left(\gamma_{1}\right)^{m}}\left(\frac{\gamma_{1} \Omega_{h_{0}}}{\Omega_{h_{1}} \Delta\left(\gamma_{1}\right)}\right)^{\frac{1-m}{2}} K_{1-m}\left(2 \sqrt{\frac{\gamma_{1} n}{\Omega_{h_{1}} \Omega_{h_{0}} \Delta\left(\gamma_{1}\right)}}\right)\right] \\
& \times\left[1-\sum_{m=0}^{M-1} \frac{\theta_{1}^{m} e^{-\frac{\theta_{1}}{\Gamma(m+1) \Omega_{1}}}}{\Gamma U_{S}}\right] .
\end{aligned}
$$

Next, the OP of $U_{2}$ is:

$$
\mathcal{O} \mathcal{P}_{U_{2}}^{\mathcal{I I}}=\operatorname{Pr}\left(\gamma_{2}^{S C}<\gamma_{2}\right)=\operatorname{Pr}\left(\gamma_{S U_{2}}<\gamma_{2}\right) \operatorname{Pr}\left(\gamma_{2,2}<\gamma_{2}\right)
$$

Similarly, $\mathcal{O} \mathcal{P}_{U_{2}}^{\mathcal{I I}}$ can be achieved as:

$$
\begin{aligned}
& \mathcal{O} \mathcal{P}_{U_{2}}^{\mathcal{I I}}=\left[1-2 \sum_{k=0}^{K-1} \sum_{n=1}^{N}\left(\begin{array}{c}
N \\
n
\end{array}\right) \frac{n \theta_{2}{ }^{k}(-1)^{n-1}}{\Gamma(m+1) \Omega_{h_{2}}^{k} \Omega_{h_{0}} \beta^{2 k}} \times\left(\frac{\theta_{2} \Omega_{h_{0}}}{\Omega_{h_{2}} \beta^{2} n}\right)^{\frac{1-k}{2}} K_{1-k}\left(2 \sqrt{\frac{\theta_{2} n}{\Omega_{h_{2}} \beta^{2} \Omega_{h_{0}}}}\right)\right] \\
& \times\left[1-e^{-\frac{\theta}{\Omega_{S U_{2}}}} \sum_{k=0}^{K-1} \frac{\theta^{k}}{\Gamma(k+1) \Omega_{S U_{2}}^{k}}\right] .
\end{aligned}
$$

\subsection{Scheme 3: outage probability of directed link by maximal ratio combining mode}

In this scheme, the backscattering link signals and direct link signals are combined by maximal ratio combining (MRC) at the two users. Hence, after using MRC the received SINR two users are given by:

$$
\gamma_{U_{i}}^{M R C}=\gamma_{S U_{i}}+\gamma_{i, i} \quad, i \in\{1,2\}
$$

Then, the OP at $U_{1}$ can be calculated as:

$$
\begin{gathered}
\mathcal{O} \mathcal{P}_{U_{1}}^{\mathcal{I} \mathcal{I} \mathcal{I}}=\operatorname{Pr}\left(\gamma_{S U_{1}}<\gamma_{1}-\gamma_{1,1}\right) \\
=\int_{0}^{\gamma_{1}} f_{\gamma_{\gamma_{1,1}}}(x) \int_{0}^{\gamma_{1}-x} f_{\gamma_{S U_{1}}}(y) d y d x .
\end{gathered}
$$

It is very difficult to obtain the close-form solution of the integral of (24). Therefore, we use the approach in [25] by making an I-step staircase approximation to the actual triangular integral region in 24] with sufficiently large I to obtain:

$$
\begin{aligned}
\mathcal{O} \mathcal{P}_{U_{1}}^{\mathcal{I I}} & \approx \sum_{i=0}^{I-1}\left(F_{\gamma_{S U_{1}}}\left(\frac{i+1}{I} \gamma_{1}\right)-F_{\gamma_{S U_{1}}}\left(\frac{i}{I} \gamma_{1}\right)\right) \\
& \times\left(F_{\gamma_{1,1}}\left(\frac{I-i}{I} \gamma_{1}\right)\right) .
\end{aligned}
$$

Thus, the OP at $U_{2}$ is given by:

$$
\begin{aligned}
\mathcal{O} \mathcal{P}_{U_{2}}^{\mathcal{I I I}} & =\operatorname{Pr}\left(\gamma_{U_{2}}^{M R C}<\gamma_{2}\right) \\
& =\int_{0}^{\theta_{2}} f_{\left\|\mathbf{h}_{S U_{2}}\right\|^{2}}(x) \bar{F}_{\left\|\mathbf{h}_{2}\right\|^{2}\left|h_{0, n^{*}}\right|^{2}}\left(\frac{\theta_{2}}{\beta^{2}}-\frac{x}{\beta^{2}}\right) d x \\
& =\frac{1}{\Gamma(K) \Omega_{h_{S U_{2}}}^{K}}\left[\Upsilon_{1}-\Upsilon_{2}\right]
\end{aligned}
$$


where $\theta_{2}=\frac{\gamma_{2}}{\rho a_{2}} . \Upsilon_{1}$ can be calculated as:

$$
\Upsilon_{1}=\int_{0}^{\theta_{2}} x^{K-1} e^{-\frac{x}{\Omega_{h_{S U_{2}}}}} d x=\Omega_{h_{S U_{2}}^{K}}^{K}\left(K, \frac{\theta_{2}}{\Omega_{h_{S U_{2}}}}\right),
$$

where $\gamma(\mathrm{x}, \mathrm{y})$ is the lower incomplete Gamma function [24], (3.351.1). And $\Upsilon_{2}$ is written as:

$$
\begin{aligned}
\Upsilon_{2}= & 2 \sum_{n=1}^{N} \sum_{k=0}^{K-1}\left(\begin{array}{c}
N \\
n
\end{array}\right) \frac{n^{(k+1) / 2}(-1)^{n-1}}{\Gamma(k+1) \Omega_{h_{0}}^{(k+1) / 2} \Omega_{h_{2}}^{(k+1) / 2}} \\
& \times \int_{0}^{\theta_{2}} x^{K-1} e^{-\frac{x}{\Omega_{h_{S U_{2}}}}\left(\frac{\theta_{2}}{\beta^{2}}-\frac{x}{\beta^{2}}\right)^{(k+1) / 2}} \\
& \times K_{1-k}\left(2 \sqrt{\frac{n}{\Omega_{h_{0}} \Omega_{h_{2}}}\left(\frac{\theta_{2}}{\beta^{2}}-\frac{x}{\beta^{2}}\right)}\right) d x,
\end{aligned}
$$

Putting $t=2 x / \theta_{2}-1 \Rightarrow \theta_{2}(t+1) / 2=x \Rightarrow \theta_{2} / 2 d t=d x$. But it is very difficult to obtain the close-form solution of $\Upsilon_{2}$, to do so, we use Gaussian-Chebyshev quadrature [26], (25.4.38), to get:

$$
\begin{aligned}
& \Upsilon_{2} \approx \sum_{n=1}^{N} \sum_{k=0}^{K-1} \sum_{l=1}^{L}\left(\begin{array}{c}
N \\
n
\end{array}\right) \frac{n^{(k+1) / 2}(-1)^{n-1} \theta_{2}}{\Omega_{h_{0}}^{(k+1) / 2} \Omega_{h_{2}}^{(k+1) / 2} 2^{K-1}} \frac{\pi \sqrt{1-\xi_{l}^{2}}\left(\xi_{l}+1\right)^{K-1} \Lambda\left(\xi_{l}\right)^{(k+1) / 2}}{\Gamma(K) \Omega_{h_{S U_{2}}}^{K} \Gamma(k+1) L} \\
& \times K_{1-k}\left(2 \sqrt{\frac{n \Lambda\left(\xi_{l}\right)}{\Omega_{h_{0}} \Omega_{h_{2}}}}\right) .
\end{aligned}
$$

Substituting (26) and (28) into (25), $\mathcal{O} \mathcal{P}_{U_{2}}^{\mathcal{I} \mathcal{I} \mathcal{I}}$ is given by:

$$
\begin{aligned}
& \mathcal{O} \mathcal{P}_{U_{2}}^{\mathcal{I} \mathcal{I}} \approx \frac{\gamma\left(K, \theta_{2} / \Omega_{h_{S U_{2}}}\right)}{\Gamma(K)}-\sum_{n=1}^{N} \sum_{k=0}^{K-1} \sum_{l=1}^{L}\left(\begin{array}{c}
N \\
n
\end{array}\right) \\
& \times \frac{n^{(k+1) / 2}(-1)^{n-1} \theta_{2} K}{\Omega_{h_{0}}^{(k+1) / 2} \Omega_{h_{2}}^{(k+1) / 2} 2^{K-1} K_{1-k}\left(2 \sqrt{\frac{n \Lambda\left(\xi_{l}\right)}{\Omega_{h_{0}} \Omega_{h_{2}}}}\right)} \\
& \times \frac{\pi \sqrt{1-\xi_{l}^{2}}\left(\xi_{l}+1\right)^{K-1} \Lambda\left(\xi_{l}\right)^{(k+1) / 2}}{\Gamma(K) \Omega_{h_{S U_{2}}}^{K} \Gamma(k+1) L} e^{-\frac{\theta_{2}\left(\xi_{l}+1\right)}{2 \Omega_{h_{S U}}}}
\end{aligned}
$$

where $\xi_{l}=\cos \left(\frac{2 l-1}{2 L} \pi\right)$ and $\Lambda(x)=\left(\frac{\theta_{2}}{\beta^{2}}-\frac{\theta_{2}(x+1)}{2 \beta^{2}}\right)$.

\section{NUMERICAL RESULTS}

In this section, we set $d_{0}=d_{1}=d_{S U_{1}}=d_{S U_{2}}=1, d_{2}=2, \Omega_{h_{0}}=d_{0}^{-\tau}, \Omega_{h_{1}}=d_{1}^{-\tau}, \Omega_{h_{2}}=d_{2}^{-\tau}$, $\Omega_{S U_{1}}=d_{S U_{1}}^{-\tau}$ and $\Omega_{S U_{1}}=d_{S U_{1}}^{-\tau}$ and $\tau$ is the path-loss exponent setting to be $\tau=2$. In particular, main parameters can be seen in Table 1. In addition, the Gauss-Chebyshev parameter is selected as $L=100$ to yield a close approximation.

Table 1. System parameters used in the performance evaluation

\begin{tabular}{cc}
\hline System Parameters & Values \\
\hline Monte Carlo simulations repeated & $10^{7}$ iterations \\
The power allocation coefficients & $\left\{a_{1}, a_{2}\right\}=\{0.8,0.2\}$ \\
The target rate at $U_{1}$ & $R_{1}=2 \mathrm{bps} / \mathrm{Hz}$ \\
The target rate at $U_{2}$ & $R_{2}=2 \mathrm{bps} / \mathrm{Hz}$ \\
The complex reflection coefficient & $\beta=0.5$ \\
\hline
\end{tabular}

Figure 2 plots OP and base station transmit SNR relationship, with with varying $N=K=M=1$ and $N=K=M=3$. In (8), (12), (19), (20), (23), and (29) are used to plot the analytical lines. From Figure 2, we notice that the network users experience different outage performances depending on the $N=K=M$ values. The best performances are achieved by scheme 3, with the worst performance obtained by scheme 1 . We can see the analytical curves fit with Monte Carlo simulations. 
Figure 3 plots the OP relationship between outage and base station transmit SNR, with varying $\beta=0.3$ and $\beta=0.7$. As in Figure 2, from Figure 3, we also observe that the users experience different outage performances depending on the complex reflection coefficient $\beta$. The best performances are achieved by scheme 3, with the worst performance obtained by user 2 in scheme 1 .

Figure 4 plots the relationship between OP and $\gamma_{1}=\gamma_{1}(\mathrm{~dB})$, varying $\beta=0.3$, and $\beta=0.7$. From Figure 4, we observe that some network users performance curves approach an outage probability ceiling. Generally, the outage performance reduces with increase in $\gamma_{1}=\gamma_{1}(\mathrm{~dB})$. The best performances are achieved by scheme 3 , with the worst performance obtained by scheme 1 .

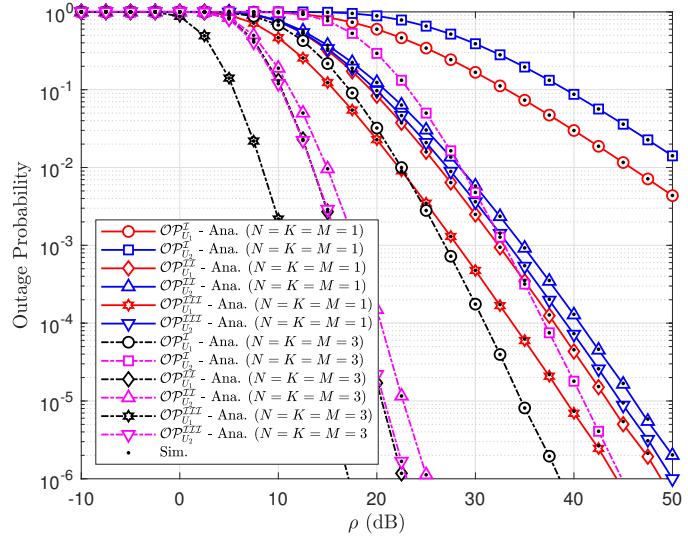

Figure 2. Outage probability versus $\rho(\mathrm{dB})$ varying $N=K=M=1$ and $N=K=M=3$

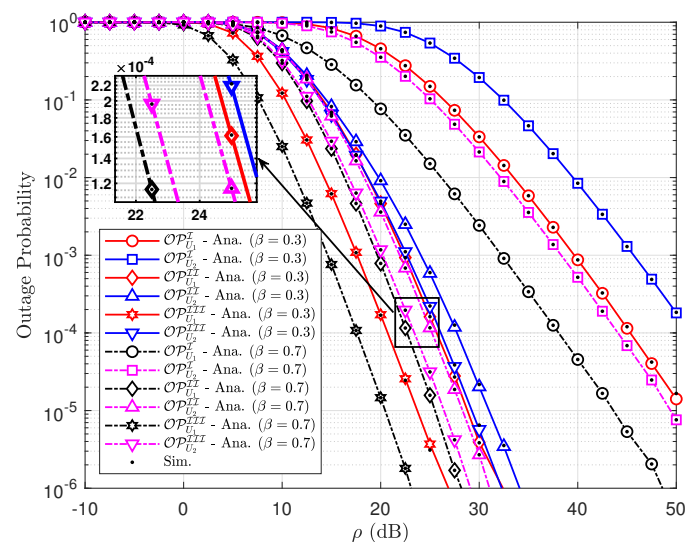

Figure 3. Outage probability versus $\rho(\mathrm{dB})$ varying $\beta=0.3$ and $\beta=0.7$

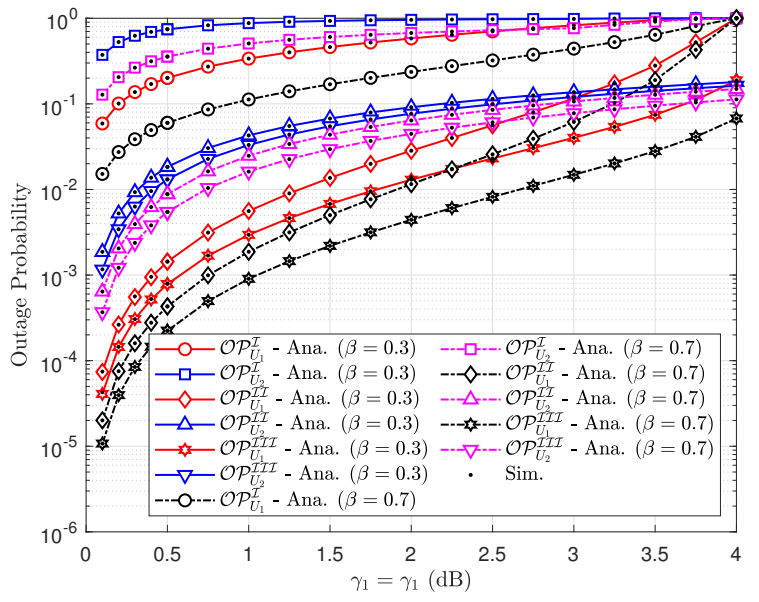

Figure 4. Outage probability versus $\gamma_{1}=\gamma_{1}(\mathrm{~dB})$ varying $\beta=0.3$ and $\beta=0.7$

Figure 5 plots the relationship between OP and power allocation coefficienct $a_{2}$, varying $N=K=$ $M=1$ and $N=K=M=2$ with $\rho=20(\mathrm{~dB})$ and $R_{1}=R_{2}=1$. From Figure 5, we note that several OP curves approach an OP floor with increasing $a_{2}$, while other OP curves converge at 0 when $a_{2}=1$. Figure 6 plots the relationship between $\mathrm{OP}$ and $N=M=K$, with $\rho=5(\mathrm{~dB})$. From Figure 5, we observe that maximal ratio combining delivers the best outage performance with the OP for backscatter-NOMA system performing the worst. In both Figures, user 2 in scheme 1 had the worst OP performance, due to the weak backscatter device signals that it recovers, therefore, it can easily experience outage in any condition. With user 1 in scheme 3 achieving the best performance due to the signals from the very near multi-backscattering devices and the further away base station direct link being combined by maximal ratio combining at its receiver. 


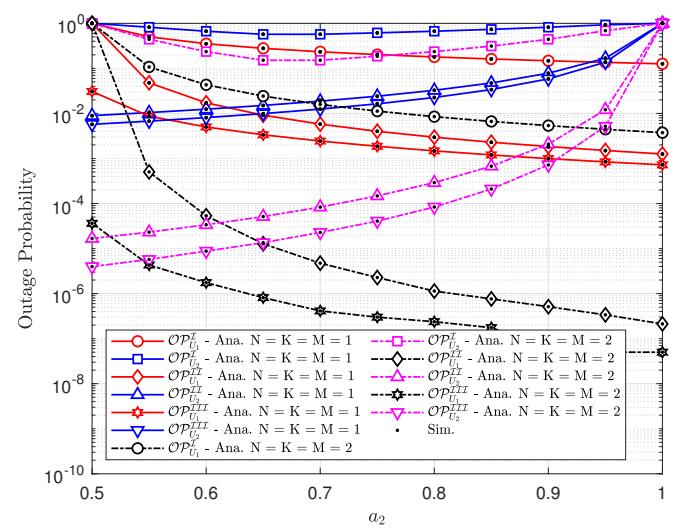

Figure 5. Outage probability versus $a_{2}$ varying $N=K=M=1$ and $N=K=M=2$ with $\rho=20$ (dB) and $R_{1}=R_{2}=1$

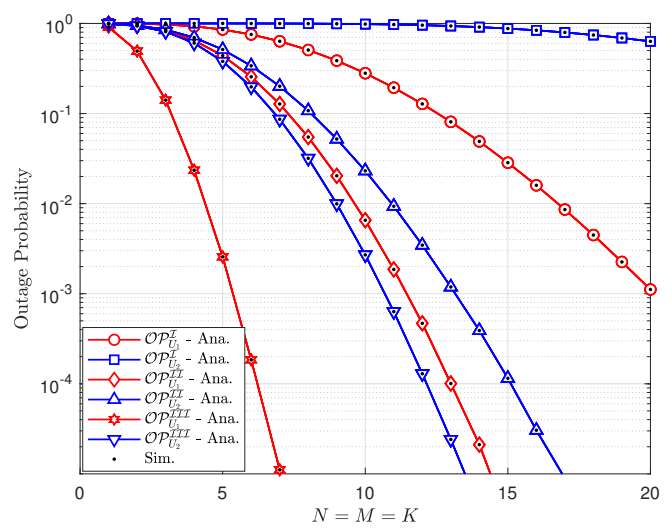

Figure 6. Outage probability versus $N=M=K$ with $\rho=5(\mathrm{~dB})$

\section{CONCLUSION}

In this paper, we provided the outage probability (OP) analysis of an ambient multi-backscatter system with multi-modes of operation. We derived exact solutions of OP for different users depending on the mode of operation. We observe from the simulation results, that the direct link between the base station and users affects the OP. In future work, we will consider ergodic capacity of the system.

\section{REFERENCES}

[1] H. Jayakumar, K. Lee, W.S. Lee, A. Raha, Y. Kim, and V. Raghunathan, "Powering the internet of things," in Proceedings of the 2014 international symposium on Low power electronics and design, pp. 375-380, 2014, doi: 10.1145/2627369.2631644.

[2] F. Jameel, S. Zeb, W. U. Khan, S. A. Hassan, Z. Chang, and J. Liu, ”NOMA-Enabled Backscatter Communications: Toward BatteryFree IoT Networks," in IEEE Internet of Things Magazine, vol. 3, no. 4, pp. 95-101, 2020, doi: 10.1109/IOTM.0001.2000055.

[3] N. Van Huynh, D. T. Hoang, X. Lu, D. Niyato, P. Wang, and D. I. Kim, "Ambient Backscatter Communications: A Contemporary Survey," in IEEE Commun. Surv. \& Tutor, vol. 20, no. 4, pp. 2889-2922, 2018, doi: 10.1109/COMST.2018.2841964.

[4] G. Yang, Y. -C. Liang, R. Zhang, and Y. Pei, "Modulation in the Air: Backscatter Communication Over Ambient OFDM Carrier," in IEEE Transactions on Communications, vol. 66, no. 3, pp. 1219-1233, March 2018, doi: 10.1109/TCOMM.2017.2772261.

[5] W. Zhao, G. Wang, R. Fan, L. Fan, and S. Atapattu, "Ambient Backscatter Communication Systems: capacity and Outage Performance Analysis," in IEEE Access, vol. 6, pp. 22695-22704, 2018, doi: 10.1109/ACCESS.2018.2828021.

[6] G. Yang, Q. Zhang, and Y. Liang, "Cooperative Ambient Backscatter Communications for Green Internet-of-Things," in IEEE Internet of Things Journal, vol. 5, no. 2, pp. 1116-1130, April 2018, doi: 10.1109/JIOT.2018.2799848.

[7] Q. Zhang, L. Zhang, Y. Liang, and P. Kam, ”Backscatter-NOMA: A Symbiotic System of Cellular and Internet-of-Things Networks,” in IEEE Access, vol. 7, pp. 20000-20013, 2019, doi: 10.1109/ACCESS.2019.2897822.

[8] B. Lyu, Z. Yang, G. Gui, and Y. Feng, "Wireless Powered Communication Networks Assisted by Backscatter Communication," in IEEE Access, vol. 5, pp. 7254-7262, 2017, doi: 10.1109/ACCESS.2017.2677521. 
[9] I. F. Akyildiz, A. Kak, and S. Nie, "6G and Beyond: the Future of Wireless Communications Systems," in IEEE Access, vol. 8, pp. 133995-134030, 2020, doi: 10.1109/ACCESS.2020.3010896.

[10] M. Z. Chowdhury, M. Shahjalal, S. Ahmed, and Y. M. Jang, "6G Wireless Communication Systems: Applications, Requirements, Technologies, Challenges, and Research Directions," in IEEE Open Journal of the Communications Society, vol. 1, pp. 957-975, 2020, doi: 10.1109/OJCOMS.2020.3010270.

[11] J. D. Griffin and G. D. Durgin, "Complete Link Budgets for Backscatter-Radio and RFID Systems," in IEEE Antennas and Propagation Magazine, vol. 51, no. 2, pp. 11-25, April 2009, doi: 10.1109/MAP.2009.5162013.

[12] C. Boyer and S. Roy, "- Invited Paper - Backscatter Communication and RFID: Coding, Energy, and MIMO Analysis," in IEEE Transactions on Communications, vol. 62, no. 3, pp. 770-785, March 2014, doi: 10.1109/TCOMM.2013.120713.130417.

[13] D. Darsena, G. Gelli, and F. Verde, "Modeling and Performance Analysis of Wireless Networks With Ambient Backscatter Devices," in IEEE Transactions on Communications, vol. 65, no. 4, pp. 1797-1814, April 2017, doi: 10.1109/TCOMM.2017.2654448.

[14] A. Khaleghi, A. Hasanvand, and I. Balasingham, "Radio Frequency Backscatter Communication for High Data Rate Deep Implants," in IEEE Transactions on Microwave Theory and Techniques, vol. 67, no. 3, pp. 1093-1106, March 2019, doi: 10.1109/TMTT.2018.2886844

[15] Dinh-Thuan Do, M. -S. Van Nguyen, M. Voznak, A. Kwasinski and J. N. de Souza, "Performance Analysis of Clustering Car-Following V2X System with Wireless Power Transfer and Massive Connections," in IEEE Internet of Things Journal, doi: 10.1109/JIOT.2021.3070744.

[16] Dinh-Thuan Do and M.-S. Van Nguyen, "Device-to-device transmission modes in NOMA network with and without Wireless Power Transfer," Computer Communications, vol. 139, pp. 67-77, May 2019, doi: 10.1016/j.comcom.2019.04.003.

[17] Dinh-Thuan Do, C. -B. Le, and F. Afghah, "Enabling Full-Duplex and Energy Harvesting in Uplink and Downlink of Small-Cell Network Relying on Power Domain Based Multiple Access," in IEEE Access, vol. 8, pp. 142772-142784, 2020, doi: 10.1109/ACCESS.2020.3013912.

[18] W. U. Khan, X. Li, M. Zeng and O. A. Dobre, "Backscatter-Enabled NOMA for Future 6G Systems: A New Optimization Framework Under Imperfect SIC," in IEEE Communications Letters, vol. 25, no. 5, pp. 1669-1672, May 2021. doi: 10.1109/LCOMM.2021.3052936

[19] Chi-Bao Le, Dinh-Thuan Do, X. Li, Y. -F. Huang, H. -C. Chen, and M. Voznak, ”Enabling NOMA in Backscatter Reconfigurable Intelligent Surfaces-Aided Systems," in IEEE Access, vol. 9, pp. 33782-33795, 2021, doi: 10.1109/ACCESS.2021.3061429.

[20] Y. Chen, "Performance of Ambient Backscatter Systems Using Reconfigurable Intelligent Surface," in IEEE Communications Letters, vol. 25, no. 8, pp. 2536-2539, Aug. 2021, doi: 10.1109/LCOMM.2021.3083110.

[21] M. Nemati, J. Ding, and J. Choi, "Short-Range Ambient Backscatter Communication Using Reconfigurable Intelligent Surfaces," 2020 IEEE Wireless Communications and Networking Conference (WCNC), 2020, pp. 1-6. doi: 10.1109/WCNC45663.2020.9120813.

[22] R. Zhang and C.-K. Ho, "MIMO broadcasting for simultaneous wireless information and power transfer," IEEE Trans. Wireless Commun., vol. 12, no. 5, pp. 1989-2001, May 2013, doi: 10.1109/TWC.2013.031813.120224.

[23] W. Huang, H. Chen, Y. Li, and B. Vucetic, "On the performance of multi-antenna wireless-powered communications with energy beamforming," IEEE Trans. on Vehicular Technology, vol. 65, no. 3, pp. 1801-1808, March 2016, doi: 10.1109/TVT.2015.2412532.

[24] I. S. Gradshteyn and I. M. Ryzhik, Table of Integrals, Series and Products, 6th ed. New York, NY, USA: Academic Press, 2000.

[25] C. Zhang, J. Ge, J. Li, Y. Rui, and M. Guizani, ”A unified approach for calculating outage performance of two-way AF relaying over fading channels," IEEE Trans. Veh. Technol., vol. 64, no. 3, pp. 1218-1229, 2015, , doi: 10.1109/TVT.2014.2329853.

[26] M. Abramowitz and I. A. Stegun, Handbook of Mathematical Functions with Formulas, Graphs, and Mathematical Tables. New York, NY, USA: Dover, 1972.

\section{BIOGRAPHIES OF AUTHORS}

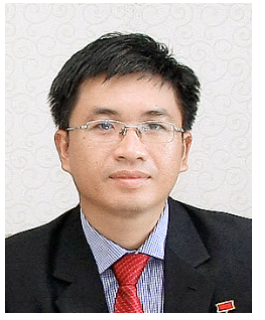

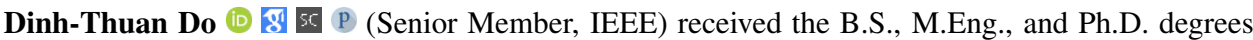
in communications engineering from Vietnam National University (VNU-HCM), in 2003, 2007, and 2013, respectively. His research interests include signal processing in wireless communications networks, cooperative communications, nonorthogonal multiple access, full-duplex transmission, and energy harvesting. He was a recipient of the Golden Globe Award from the Vietnam Ministry of Science and Technology, in 2015 (Top ten excellent young scientists nationwide). He has served as a guest editor for eight prominent SCIE journals. He is currently serving as an associate editor for five journals, including EURASIP Journal on Wireless Communications and Networking, Computer Communications (Elsevier), ICT Express, Electronics and KSII Transactions on Internet and Information Systems. He can be contacted at email: dodinhthuan@iuh.edu.vn.

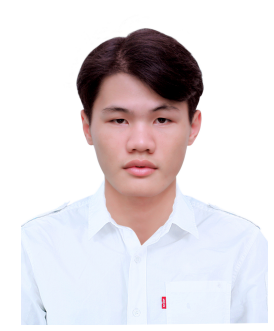

Anh-Tu Le 10 (8) 5 (Member, IEEE) was born in Lam Dong, Vietnam, in 1997. He is pursuing the Ph.D. degree in communication and information system the field of wireless communication. He is currently a Research Assistant with the WICOM Laboratory, which was led by Dr. Thuan. He has authored or co-authored over 25 technical articles published in peer-reviewed international journals (SCIE). His research interests include the wireless channel modeling, NOMA, cognitive radio, and MIMO. He can be contacted at email: leanhtu@iuh.edu.vn. 